\title{
AN AVERAGING RESULT FOR PERIODIC SOLUTIONS OF CARATHÉODORY DIFFERENTIAL EQUATIONS
}

\author{
DOUGLAS D. NOVAES
}

\begin{abstract}
This paper is concerned with the problem of existence of periodic solutions for perturbative Carathéodory differential equations. The main result provides sufficient conditions on the averaged equation that guarantee the existence of periodic solutions. Additional conditions are also provided to ensure the uniform convergence of a periodic solution to a constant function. The proof of the main theorem is mainly based on an abstract continuation result for operator equations.
\end{abstract}

\section{INTRODUCTION AND STATEMENT OF THE MAIN RESULT}

This paper is concerned with the problem of existence of periodic solutions for differential equations given in the following standard form:

$$
x^{\prime}=\varepsilon f(t, x, \varepsilon), \quad(t, x, \varepsilon) \in \mathbb{R} \times D \times\left[0, \varepsilon_{0}\right],
$$

where $D$ is an open subset of $\mathbb{R}^{n}, \varepsilon_{0}>0$, and $f: \mathbb{R} \times D \times\left[0, \varepsilon_{0}\right] \rightarrow \mathbb{R}^{n}$ is assumed to be $T$ periodic in the variable $t$. Further conditions will be assumed later on.

The averaging method is an important technique for investigating differential equations given in the standard form (1). It has a long history, starting with the development of celestial mechanics by Clairaut, Laplace, and Lagrange, which was later formalized by the works of Fatou, Krylov, Bogoliubov, and Mitropolsky [1, 2, 6, 9] (for a historical review, see [18, Chapter 6] and [20, Appendix A]). The averaging method concerns about asymptotic estimates for solutions of differential equations. More specifically, it provides conditions under which the solutions of (1) remains $\varepsilon$-close to the solutions of the truncated average equation $x^{\prime}=\varepsilon f_{1}(x)$, for an order $\varepsilon^{-1}$ interval of time. Here, assuming that, for each $z \in D$, the map $t \in \mathbb{R} \mapsto f(t, z, 0)$ is integrable and T-periodic, $f_{1}(z)$ denotes its average in the variable $t$, that is,

$$
f_{1}(z)=\frac{1}{T} \int_{0}^{T} f(t, z, 0) d t .
$$

Periodic trajectories are of major importance in the understanding of the qualitative behavior of differential equations. The averaging method is one of the most powerful technique for investigating periodic solutions of differential equations given as (1). One can find many results in the classical research literature relating isolated zeros of the averaged function $f_{1}(z)$ with $T$-periodic solutions of (1) (see, for instance, [8, 20, 21]). The aforementioned results assume smoothness of $f$. These results have been generalized in several directions: in [3, 13, 19], for nonsmooth continuous differential equations; in [11, 12, 14], for several classes of discontinuous piecewise smooth differential equations; and, in [5], for abstract semilinear equations.

2010 Mathematics Subject Classification. 34C29, 34C25, 47H11, 34A36.

Key words and phrases. Carathéodory differential equations, averaging method, periodic solutions, continuation result for operator equations. 
In this paper, it is assumed that the differential equation (1) satisfies the following Carathéodory conditions:

A.1 $(x, \varepsilon) \in D \times\left[0, \varepsilon_{0}\right] \mapsto f(t, x, \varepsilon)$ is continuous, for almost every $t \in[0, T]$;

A.2 $t \in[0, T] \mapsto f(t, x, \varepsilon)$ is measurable, for every $(x, \varepsilon) \in D \times\left[0, \varepsilon_{0}\right]$;

A.3 for each $r>0$, there exists an integrable function $g_{r}:[0, T] \rightarrow \mathbb{R}$ such that, for almost every $t \in[0, T],|f(t, x, \varepsilon)| \leq g_{r}(t)$ provided that $|(x, \varepsilon)| \leq r$.

Accordingly, (1) is called Carathéodory differential equation (see [10]).

Notice that, as consequences of the dominate convergence theorem, conditions A.2 and A.3 allow the computation of the averaged function $f_{1}(z)$ which, taking conditions A.1 and A.3 into account, is continuous on $D$.

There are some results in the research literature concerning periodic solutions for Carathéodory differential equations. In [17], an abstract continuation result for operator equations [17, Theorem IV.13], due to Mawhin [15, 16] (see also [7. Corollary IV.1]), was used to prove a guiding function method [17. Theorem VI.2] that provides sufficient conditions for the existence of periodic solutions for Carathéodory differential equations. In [4], by using an approximation procedure, [4, Theorem 1] established that the coincidence degree of an autonomous differential equation $x^{\prime}-g(x)=0$ can be computed in terms of the Brouwer degree of $g$ and, then, it was used to provide a continuation result [4, Theorem 2] for periodic solutions of Carathéodory differential systems given by $x^{\prime}=g(x)+e(t, x)$. The above studies treated the problem of existence of periodic solutions by means of topological methods.

In this paper, an abstract continuation result for operator equations, [7, Theorem IV.2], will be used to provide sufficient conditions on the averaged function $f_{1}(z)$ that guarantee the existence of periodic solutions for the Carathéodory differential equation (1). Additional conditions will be also provided to ensure that a periodic solution converges uniformly to a constant function as $\varepsilon$ goes to 0 . In what follows, $d_{B}$ denotes the Brouwer degree (the reader is referred to Section 2 for its formal definition).

Theorem A. Consider the Carathéodory differential equation (1). Assume that there exists an open bounded set $V \subset \mathbb{R}^{n}$, with $\bar{V} \subset D$, such that $f_{1}(z) \neq 0$, for every $z \in \partial V$, and $d_{B}\left(f_{1}, V, 0\right) \neq$ 0 . Then, there exists $\varepsilon_{V}>0$ such that, for each $\varepsilon \in\left[0, \varepsilon_{V}\right]$, the differential equation (1) has a $T$-periodic solution $\varphi(t, \varepsilon)$ satisfying $\varphi(t, \varepsilon) \in V$, for every $t \in[0, T]$. In addition, if there exists $z^{*} \in V$ such that $f_{1}\left(z^{*}\right)=0$ and $f_{1}(z) \neq 0$ for every $z \in \bar{V} \backslash\left\{z^{*}\right\}$, then $\varphi(\cdot, \varepsilon) \rightarrow z^{*}$, uniformly, as $\varepsilon \rightarrow 0$.

Remark 1. Under the assumptions " $z \mapsto f_{1}(z)$ is differentiable in a neighbourhood of $z=z^{*}$, $f_{1}\left(z^{*}\right)=0$, and $f_{1}(z) \neq 0$ for every $z \in \bar{V} \backslash\left\{z^{*}\right\}^{\prime \prime}$, the condition $d_{B}\left(f_{1}, V, 0\right) \neq 0$ holds provided that $\operatorname{det}\left(D f_{1}\left(z^{*}\right)\right) \neq 0$ and, in that case, $d_{B}\left(f_{1}, V, 0\right)=\operatorname{sign}\left(\operatorname{det}\left(D f_{1}\left(z^{*}\right)\right)\right)$.

Usually, the averaging method for studying periodic solutions, even for continuous differential equations, assumes a smooth dependence of $f$ on the parameter of perturbation $\varepsilon$. Also, the existence of periodic solutions strongly relies on the existence of isolated zeros of the averaged function $f_{1}$ (see, for instance, [3, Theorem 1.2] and [19. Corollary 2.2]). Here, Theorem A, in addition to extending the previous results for Carathéodory differential equations, relaxes such a smoothness assumption on $f$ and the existence of periodic solutions is provided regardless the existence of isolated zeros of $f_{1}$. Its proof, based on the degree theory, is similar to the ones performed in [19]. Here, the dominate convergence theorem is needed for defining suitable operators in order to employ an abstract continuation result for operator equations. 
The degree theory and an abstract continuation result for operator equations are presented in Section 2 Theorem A is, then, proven in Section 3 . Section 4 is devoted to investigate some examples.

\section{CONTINUATION RESUlt FOR OPERATOR EQUATIONS}

This section presents a useful abstract continuation result, Theorem 1 , which provides sufficient conditions for the existence of solutions of the operator equation

$$
L x=\varepsilon N(x, \varepsilon), x \in \bar{\Omega},
$$

where $L: \operatorname{dom} L \subset X \rightarrow Y$ is a linear map between real normed vector spaces $X$ and $Y$, dom $L$ is a subspace of $X, \varepsilon \in\left[0, \varepsilon_{0}\right]$, with $\varepsilon_{0}>0, \Omega$ is an open bounded subset of $X$ such that $\bar{\Omega} \subset \operatorname{dom} L$, and $N: \bar{\Omega} \times\left[0, \varepsilon_{0}\right] \rightarrow Y$ is any map.

In addition, it is assumed that $L$ is Fredholm of index 0 and that $N$ is $L$-compact. Following [17. Chapter I], such concepts are introduced in the sequel.

The linear map $L$ is said to be Fredholm of index 0 if $\operatorname{ker} L$ has finite dimension, $\operatorname{Im} L$ is closed in $Y$ and has finite codimension (that is coker $L=Y / \operatorname{Im} L$ has finite dimension), and the dimension of ker $L$ coincides with the codimension of $\operatorname{Im} L$. In this case, there exist continuous projections $P: X \rightarrow X$ and $Q: Y \rightarrow Y$ satisfying

$$
\operatorname{Im} P=\operatorname{ker} L \text { and } \operatorname{ker} Q=\operatorname{Im} L \text {, }
$$

which implies that

$$
X=\operatorname{ker} L \oplus \operatorname{ker} P \text { and } Y=\operatorname{Im} L \oplus \operatorname{Im} Q .
$$

Notice that $\operatorname{Im} Q$ is isomorphic to coker $L$ and, consequently, isomorphic to ker $L$. In addition, one can see that $L_{P}=\left.L\right|_{\operatorname{ker} P \cap \operatorname{dom} L}$ is an isomorphism onto $\operatorname{Im} L$. Accordingly, denote

$$
K_{P}=L_{P}^{-1}: \operatorname{Im} L \rightarrow \operatorname{ker} P \cap \operatorname{dom} L
$$

and define $K_{P, Q}=K_{P}(I d-Q)$.

The map $N: \bar{\Omega} \times\left[0, \varepsilon_{0}\right] \rightarrow Y$ is said to be $L$-compact on $\bar{\Omega} \times\left[0, \varepsilon_{0}\right]$ if the maps $K_{P, Q} N$ : $\bar{\Omega} \times\left[0, \varepsilon_{0}\right] \rightarrow X$ and $Q N: \bar{\Omega} \times\left[0, \varepsilon_{0}\right] \rightarrow Y$ are continuous and the subsets $K_{P, Q} N(\bar{\Omega} \times$ $\left.\left[0, \varepsilon_{0}\right]\right)$ and $Q N\left(\bar{\Omega} \times\left[0, \varepsilon_{0}\right]\right)$ are relatively compact on $X$ and $Y$, respectively. It can be seen that the definition of $L$-compactness does not depend on the choices of $P$ and $Q$ satisfying (3).

Theorem 1 ([7, Theorem IV.2]). Let $L, N$, and $Q$ be like above and $J: \operatorname{Im} Q \rightarrow \operatorname{ker} L$ any isomorphism. Assume that the following conditions are verified:

H.1 $Q N(x, 0) \neq 0$, for every $x \in \partial \Omega \cap$ ker $L$; and

H.2 $d_{B}\left(\left.J Q N(\cdot, 0)\right|_{\Omega \cap \operatorname{ker} L^{\prime}} \Omega \cap \operatorname{ker} L, 0\right) \neq 0$.

Then, there exists $\varepsilon_{1} \in\left(0, \varepsilon_{0}\right]$ such that, for each $\varepsilon \in\left[0, \varepsilon_{1}\right]$, the operator equation (2) admits a solution in $\Omega$.

Theorem 1 makes use of the concept of Brouwer degree $d_{B}$, which is defined as follows (see [22]). Let $V \subset \mathbb{R}^{n}$ be an open bounded subset of $\mathbb{R}^{n}, g: \bar{V} \rightarrow \mathbb{R}^{n}$ a continuous function, and $y_{0} \notin g(\partial V)$. The Brouwer degree $d_{B}\left(g, V, y_{0}\right)$ is characterized as the unique integer-valued function satisfying the following properties:

P.1 If $d_{B}\left(g, V, y_{0}\right) \neq 0$, then $y_{0} \in g(V)$. Furthermore, if $\mathbb{1}: \bar{V} \rightarrow \mathbb{R}^{n}$ is the identity function and $y_{0} \in V$, then $d_{B}\left(\mathbb{1}, V, y_{0}\right)=1$.

P.2 If $V_{1}, V_{2} \subset V$ are disjoint open subsets of $V$ such that $y_{0} \notin g\left(\bar{V} \backslash\left(V_{1} \cup V_{2}\right)\right)$, then

$$
d_{B}\left(g, V, y_{0}\right)=d_{B}\left(\left.g\right|_{V_{1}}, V_{1}, y_{0}\right)+d_{B}\left(\left.g\right|_{V_{2}}, V_{2}, y_{0}\right) \text {. }
$$


D.D. NOVAES

P.3 If $\left\{g_{\sigma}: \bar{V} \rightarrow \mathbb{R}^{n} \mid \sigma \in[0,1]\right\}$ is a continuous homotopy and $\left\{y_{\sigma} \mid \sigma \in[0,1]\right\}$ is a continuous curve in $Y$ such that $y_{\sigma} \notin g_{\sigma}(\partial V), \forall \sigma \in[0,1]$ then $d_{B}\left(g_{\sigma}, V, y_{\sigma}\right)$ is constant for $\sigma \in[0,1]$.

\section{Proof of Theorem $\mathrm{A}$}

The proof of Theorem A will follow directly from Propositions 1 and 2

Proposition 1. Consider the Carathéodory differential equation (1). Assume that there exists an open bounded set $V \subset \mathbb{R}^{n}$, with $\bar{V} \subset D$, such that $f_{1}(z) \neq 0$, for every $z \in \partial V$, and $d_{B}\left(f_{1}, V, 0\right) \neq$ 0 . Then, there exists $\varepsilon_{V}>0$ such that, for each $\varepsilon \in\left[0, \varepsilon_{V}\right]$, the differential equation (1) has a $T$-periodic solution $\varphi(t, \varepsilon)$ satisfying $\varphi(t, \varepsilon) \in V$, for every $t \in[0, T]$.

Proof. The first step of this proof consists in establishing suitable spaces and operators for applying Theorem 1 It is worth mentioning that the initial framework of this proof is similar to the proof of [19, Theorem A].

Consider the following real Banach spaces

$$
X=\left\{x \in C\left([0, T], \mathbb{R}^{n}\right): x(0)=x(T)\right\} \text { and } Y=\left\{x \in C\left([0, T], \mathbb{R}^{n}\right): x(0)=0\right\},
$$

and let $\Omega$ be the following open bounded subset of $X$,

$$
\Omega=\{x \in X: x(t) \in V, \forall t \in[0, T]\} .
$$

As usual, $C\left([0, T], \mathbb{R}^{n}\right)$ denotes the space of continuous functions $x:[0, T] \rightarrow \mathbb{R}^{n}$ endowed with the sup-norm.

Define the linear map $L: X \rightarrow Y$ by

$$
L x(t)=x(t)-x(0)
$$

and let $N: \bar{\Omega} \times\left[0, \varepsilon_{0}\right] \rightarrow Y$ be given by

$$
N(x, \varepsilon)(t)=\int_{0}^{t} f(s, x(s), \varepsilon) d s .
$$

In order to see that $N$ is well defined, let $r_{V}>0$ satisfy $|(x(t), \varepsilon)|<r_{V}$ for every $(t, x, \varepsilon) \in$ $[0, T] \times \bar{\Omega} \times\left[0, \varepsilon_{0}\right]$. From condition A.3, there exists an integrable function $g_{r_{V}}:[0, T] \rightarrow \mathbb{R}$ satisfying $|f(t, x, \varepsilon)| \leq g_{r_{V}}(t)$ for every $(x, \varepsilon) \in \bar{V} \times\left[0, \varepsilon_{0}\right]$ and for almost every $t \in[0, T]$. Thus, taking conditions A.2 and A.3 into account, it follows that the integral $N(x, \varepsilon)(t)$ is well defined for each $(x, \varepsilon) \in \bar{\Omega} \times\left[0, \varepsilon_{0}\right]$ and for every $t \in[0, T]$. In addition, consider the function $G_{r_{V}}:[0, T] \rightarrow \mathbb{R}$ given by

$$
G_{r_{V}}(t)=\int_{0}^{t} g_{r_{V}}(s) d s
$$

From the dominate convergence theorem, $G_{r_{V}}$ is continuous on $[0, T]$ and, therefore, uniformly continuous. In addition, one can see that

$$
|N(x, \varepsilon)(t)| \leq G_{r_{V}}(t)
$$

for every $t \in[0, T]$, and

$$
|N(x, \varepsilon)(t)-N(x, \varepsilon)(\tau)| \leq\left|G_{r_{V}}(t)-G_{r_{V}}(\tau)\right|,
$$

for every $t, \tau \in[0, T]$. The relationship (4) implies that $N(x, \varepsilon)(0)=0$. The relationship (5) and the uniform continuity of $G_{r_{V}}$ imply that $t \mapsto N(x, \varepsilon)(t)$ is continuous. Hence, $N(x, \varepsilon) \in Y$ for every $(x, \varepsilon) \in \bar{\Omega} \times\left[0, \varepsilon_{0}\right]$, which implies that $N$ is well defined. 
Now, given $\left(x^{*}, \varepsilon^{*}\right) \in \bar{\Omega} \times\left[0, \varepsilon_{0}\right]$, consider a sequence $\left(x_{n}, \varepsilon_{n}\right) \in \bar{\Omega} \times\left[0, \varepsilon_{0}\right]$ such that $\left(x_{n}, \varepsilon_{n}\right) \rightarrow\left(x^{*}, \varepsilon^{*}\right)$. For $t \in[0, T]$, define

$$
\Delta_{n}(t)=f\left(t, x^{*}(t), \varepsilon^{*}\right)-f\left(t, x_{n}(t), \varepsilon_{n}\right)
$$

Notice that

$$
\left|N\left(x^{*}, \varepsilon^{*}\right)-N\left(x_{n}, \varepsilon_{n}\right)\right| \leq \int_{0}^{T}\left|\Delta_{n}(s)\right| d s .
$$

Since, for almost every $t \in[0, T], \lim \Delta_{n}(t)=0$ (by A.1) and $\left|\Delta_{n}(t)\right| \leq 2 g_{r_{V}}(t)$ (by A.3), again from the dominate convergence theorem, one has that

$$
\lim \left|N\left(x^{*}, \varepsilon^{*}\right)-N\left(x_{n}, \varepsilon_{n}\right)\right|=\lim \int_{0}^{T}\left|\Delta_{n}(s)\right| d s=0,
$$

which implies that $N$ is continuous.

Now, consider the operator equation

$$
L x=\varepsilon N(x, \varepsilon), x \in \bar{\Omega} .
$$

Notice that $x \in \bar{\Omega}$ is a solution of $(6)$ if, and only if, it can can be continued to a $T$-periodic solution of the differential equation (1) in $\bar{V}$. (6).

In what follows, the conditions of Theorem 1 will be verified for the operator equation

First, notice that $\operatorname{Im} L=X \cap Y$ is closed in $Y$ and

$$
\text { ker } L=\left\{x \in X: x(t)=z \in \mathbb{R}^{n}, \forall t \in[0, T]\right\}
$$

is isomorphic to $\mathbb{R}^{n}$. It is easy to see that coker $L$ is also isomorphic to $\mathbb{R}^{n}$. Hence, $L$ is Fredholm of index 0 .

In order to see that $N$ is $L$-compact on $\bar{\Omega} \times\left[0, \varepsilon_{0}\right]$, let $P: X \rightarrow X$ and $Q: Y \rightarrow Y$ be continuous projections given, respectively, by

$$
P x(t)=x(0) \text { and } Q y(t)=t \frac{y(T)}{T}, \text { for } t \in[0, T] .
$$

Notice that Property (3) is satisfied for $P$ and $Q$. Clearly, $K_{P, Q} N$ and $Q N$ are continuous. Moreover, taking into account (4), (5), and the Arzelà-Ascoli Theorem, one can see that the families of continuous functions $K_{P, Q} N\left(\bar{\Omega} \times\left[0, \varepsilon_{0}\right]\right)$ and $Q N\left(\bar{\Omega} \times\left[0, \varepsilon_{0}\right]\right)$ are relatively compact on $X$ and $Y$, respectively. Hence, $N$ is $L$-compact on $\bar{\Omega} \times\left[0, \varepsilon_{0}\right]$.

Now, let $x \in \partial \Omega \cap \operatorname{ker} L$. Notice that $x(t) \equiv z \in \partial V$. Thus,

$$
Q N(x, 0)(t)=\frac{t}{T} \int_{0}^{T} f(s, z, 0) d s=t f_{1}(z)
$$

By hypothesis, $f_{1}(z) \neq 0$ for every $z \in \partial V$, so $Q N(x, 0) \neq 0$ for every for $x \in \partial \Omega \cap$ ker $L$. Therefore, condition H.1 of Theorem 1 holds for the operator equation (6).

In addition, let the isomorphism $J: \operatorname{Im} Q \rightarrow \operatorname{ker} L$ be given by

$$
J y(t)=\frac{y(T)}{T} \text {. }
$$

From (7), $J Q N(x, 0)=f_{1}(z)$, for every $x \in \Omega \cap \operatorname{ker} L$ (in this case, $x(t) \equiv z \in V$ ). Thus,

$$
d_{B}\left(\left.J Q N(\cdot, 0)\right|_{\Omega \cap \operatorname{ker} L^{\prime}} \Omega \cap \operatorname{ker} L, 0\right)=d_{B}\left(f_{1}, V, 0\right) .
$$

By hypothesis, $d_{B}\left(f_{1}, V, 0\right) \neq 0$, so condition $\mathbf{H . 2}$ holds for the operator equation 6 .

Thus, Theorem 1 provides the existence of $\varepsilon_{V} \in\left(0, \varepsilon_{0}\right]$ such that, for each $\varepsilon \in\left[0, \varepsilon_{V}\right]$, the operator equation (6) has a solution $x_{\varepsilon} \in \Omega$. Hence, for each $\varepsilon \in\left[0, \varepsilon_{V}\right], \varphi(t, \varepsilon)=x_{\varepsilon}(t)$ is a 
$T$-periodic solution of the Carathéodory differential equation (1) satisfying $\varphi(t, \varepsilon) \in V$ for every $t \in[0, T]$.

Proposition 2. In addition to hypotheses of Proposition 1 assume that there exists $z^{*} \in V$ such that $f_{1}\left(z^{*}\right)=0$ and $f_{1}(z) \neq 0$ for every $z \in \bar{V} \backslash\left\{z^{*}\right\}$. Then, for each $\varepsilon \in\left[0, \varepsilon_{V}\right]$, the differential equation (1) has a $T$-periodic solution $\varphi(t, \varepsilon)$ satisfying $\varphi(\cdot, \varepsilon) \rightarrow z^{*}$, uniformly, as $\varepsilon \rightarrow 0$.

Proof. Let $\mu_{0}>0$ satisfy $V_{\mu}:=B\left(z^{*}, \mu\right) \subset V$ for every $\mu \in\left(0, \mu_{0}\right]$.

By hypothesis, $f_{1}(z) \neq 0$, for every $x \in \partial V_{\mu}$ and $\mu \in\left(0, \mu_{0}\right]$.

Also, Properties P.1 and P.2 of the Brouwer degree imply that

$$
d_{B}\left(f_{1}, V_{\mu}, 0\right)=d_{B}\left(f_{1}, V, 0\right) \neq 0,
$$

for every $\mu \in\left(0, \mu_{0}\right]$. Indeed, consider $V^{\prime}=\operatorname{int}\left(V \backslash V_{\mu}\right)$, since $0 \notin f_{1}\left(V^{\prime}\right)$, Property P.1 implies that $d_{B}\left(f_{1}, V^{\prime}, 0\right)=0$. Now, since $0 \notin f_{1}\left(\bar{V} \backslash\left(V_{\mu} \cup V^{\prime}\right)\right)$, Property P.2 implies that

$$
d_{B}\left(f_{1}, V, 0\right)=d_{B}\left(f_{1}, V_{\mu}, 0\right)+d_{B}\left(f_{1}, V^{\prime}, 0\right)=d_{B}\left(f_{1}, V_{\mu}, 0\right) .
$$

Hence, all the hypotheses of Proposition 1 are satisfied by taking $V_{\mu}$ instead of $V$. Consequently, for each $\mu \in\left(0, \mu_{0}\right]$, there exists $\varepsilon_{V_{\mu}}>0$ such that, for each $\varepsilon \in\left[0, \varepsilon_{V_{\mu}}\right]$, the differential equation (1) has a $T$-periodic solution $\varphi^{\mu}(t, \varepsilon)$ satisfying $\varphi^{\mu}(t, \varepsilon) \in V_{\mu}$, for every $t \in[0, T]$.

Finally, a $T$-periodic solution $\varphi(t, \varepsilon)$ of the differential equation (1) converging uniformly to the constant function $z^{*}$ will be obtained from the family of periodic solutions $\left\{\varphi^{\mu}\right.$ : $\left.\mathbb{R} \times\left[0, \varepsilon_{V_{\mu}}\right] \rightarrow \mathbb{R}^{n}, \mu \in\left(0, \mu_{0}\right]\right\}$ obtained above.

Let $\bar{n} \in \mathbb{N}$ satisfy $1 / \bar{n}<\mu_{0}$. For each $n \in \mathbb{N}$, set $\mu_{n}=1 /(\bar{n}+n) \in\left(0, \mu_{0}\right]$ and denote $\varphi_{n}=\varphi^{\mu_{n}}$ and $\varepsilon_{n}=\varepsilon_{V_{\mu_{n}}} \in\left(0, \varepsilon_{0}\right]$. Notice that, for each $n \in \mathbb{N}, \varphi_{n}(t, \varepsilon) \in V_{\mu_{n}}=B\left(z^{*}, \mu_{n}\right)$ for every $t \in[0, T]$ and $\varepsilon \in\left[0, \varepsilon_{n}\right]$. Without loss of generality, one can assume that the sequence $\varepsilon_{n}$ converges to $\varepsilon^{*} \in\left[0, \varepsilon_{0}\right]$. From here, the cases $\varepsilon^{*}>0$ and $\varepsilon^{*}=0$ will be distinguished.

If $\varepsilon^{*}>0$, then there exists $\bar{\varepsilon} \in\left(0, \varepsilon_{0}\right]$ and $n_{0}>0$ such that $[0, \bar{\varepsilon}] \subset\left[0, \varepsilon_{n}\right]$ for every $n>n_{0}$. Thus, the sequence of periodic solutions $\varphi_{n}(t, \varepsilon), n>n_{0}$, satisfies $\varphi_{n}(t, \varepsilon) \in B\left(z^{*}, \mu_{n}\right)$ for every $t \in[0, T]$ and $\varepsilon \in[0, \bar{\varepsilon}]$. Thus, $\varphi_{n} \rightarrow z^{*}$ uniformly and, consequently, $\varphi(t, \varepsilon)=z^{*}$ is a periodic solutions of (1) for every $\varepsilon \in[0, \bar{\varepsilon}]$.

Otherwise, if $\varepsilon^{*}=0$, let $\varepsilon_{M}=\max \left\{\varepsilon_{n}: n \in \mathbb{N}\right\}$. Then, for each $\varepsilon \in\left(0, \varepsilon_{M}\right]$ define

$$
n_{\varepsilon}=\max \left\{n \in \mathbb{N}: \varepsilon_{n} \geq \varepsilon\right\} \text {. }
$$

Notice that $n_{\varepsilon} \rightarrow \infty$ as $\varepsilon \rightarrow 0$. Therefore, taking $\varphi(t, \varepsilon)=\varphi_{n_{\varepsilon}}(t, \varepsilon)$, one can see that $\varphi(t, \varepsilon) \in$ $B\left(z^{*}, \mu_{n_{\varepsilon}}\right)$. Hence, $\varphi(\cdot, \varepsilon) \rightarrow z^{*}$, uniformly, as $\varepsilon \rightarrow 0$.

\section{EXAMPLES}

Given $\varepsilon_{0}>0$ and $A=\left\{(x, \dot{x}) \in \mathbb{R}^{2}: r_{0} \leq \sqrt{x^{2}+\dot{x}^{2}} \leq r_{1}\right\}$, with $r_{1}>r_{0}>0$, consider the following second order differential equation

$$
\ddot{x}=-x+\varepsilon g(x, \dot{x}, \varepsilon), \quad(x, \dot{x}, \varepsilon) \in A \times\left[0, \varepsilon_{0}\right] .
$$

Assume that

$$
\tilde{g}(\theta, r, \varepsilon)=g(r \cos \theta, r \sin \theta, \varepsilon)
$$

satisfies A.1-A.3. Applying the polar change of coordinates, $(x, \dot{x})=(r \cos \theta, r \sin \theta)$, and taking $\theta$ as the new time variable, the second order differential equation (8) becomes

$$
\frac{d r}{d \theta}=\varepsilon f(\theta, r, \varepsilon)
$$


where $(\theta, r, \varepsilon) \in \mathbb{R} \times\left[r_{0}, r_{1}\right] \times\left[0, \varepsilon_{0}\right]$ and

$$
f(\theta, r, \varepsilon)=-\frac{r \tilde{g}(\theta, r, \varepsilon) \sin \theta}{r-\varepsilon \tilde{g}(\theta, r, \varepsilon) \cos \theta} .
$$

Notice that, by taking $\varepsilon_{0}>0$ smaller if necessary, $f$ also satisfies A.1-A.3. In addition,

$$
f_{1}(r)=-\frac{1}{2 \pi} \int_{0}^{2 \pi} \tilde{g}(\theta, r, 0) \sin \theta d \theta .
$$

Example 1. Suppose that $[1,2] \subset\left(r_{0}, r_{1}\right)$ and let

$$
g(x, y, 0)=\operatorname{sign}\left(y\left(x^{2}+y^{2}-1\right)\right) \max \left\{0,\left(x^{2}+y^{2}-1\right)\left(x^{2}+y^{2}-4\right)\right\} .
$$

Notice that

satisfies A.1-A.3 and

$$
\tilde{g}(\theta, r, 0)=\operatorname{sign}\left(\left(r^{2}-1\right) \sin \theta\right) \max \left\{0,\left(r^{2}-1\right)\left(r^{2}-4\right)\right\}
$$

$$
f_{1}(r)= \begin{cases}\frac{2}{\pi}\left(r^{4}-5 r^{2}+4\right), & r<1 \\ 0, & 1 \leq r \leq 2 \\ -\frac{2}{\pi}\left(r^{4}-5 r^{2}+4\right), & r>2\end{cases}
$$

Now, let $V$ be any open interval in $\left[r_{0}, r_{1}\right]$ containing $[1,2]$. It is easy to construct a homotopy $\left\{g_{\sigma}\right.$ : $\left.\bar{V} \rightarrow \mathbb{R}^{n} \mid \sigma \in[0,1]\right\}$ between $\left.f_{1}\right|_{\bar{V}}$ and $g_{1}: r \mapsto 3 / 2-r, r \in \bar{V}$, such that $0 \notin g_{\sigma}(\partial V), \forall \sigma \in$ $[0,1]$ (see Figure 1).

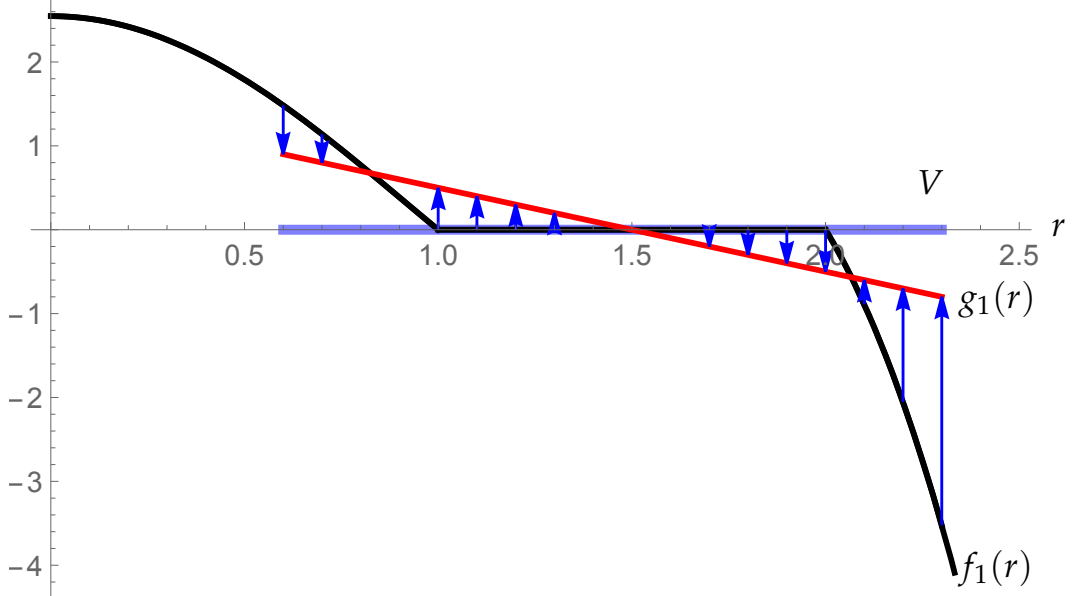

FIGURE 1. Illustration of the homotopy $g_{\sigma}$ between $f_{1}(r)$ and $g_{1}(r)=$ $3 / 2-r$. Notice that $0 \notin g_{\sigma}(\partial V), \forall \sigma \in[0,1]$.

Property $\mathbf{P} .3$ of the Brouwer degree implies

$$
d_{B}\left(f_{1}, V, 0\right)=d_{B}\left(g_{1}, V, 0\right)=-1
$$

(see Remark 1). Hence, Theorem A guarantees, for $\varepsilon>0$ sufficiently small, the existence of a periodic solution $r(\theta, \varepsilon)$ of the differential equation (9) satisfying $r(\theta, \varepsilon) \in V$ for every $\theta \in \mathbb{R}$. This corresponds to a periodic solution $(x(t, \varepsilon), \dot{x}(t, \varepsilon))$ of the second order differential equation (8) satisfying $(x(t, \varepsilon), \dot{x}(t, \varepsilon)) \in A_{V}$ for every $t \in \mathbb{R}$, where $A_{V}=\left\{(x, \dot{x}) \in \mathbb{R}^{2}: \sqrt{x^{2}+\dot{x}^{2}} \in V\right\}$. 
Example 2. Suppose that $r_{0}<1<r_{1}$ and let

$$
g(x, y, 0)=\operatorname{sign}(y) \sqrt[3]{x^{2}+y^{2}-1}
$$

Notice that

satisfies A.1-A.3 and

$$
\tilde{g}(\theta, r, 0)=\operatorname{sign}(\sin \theta) \sqrt[3]{r^{2}-1}
$$

$$
f_{1}(r)=-\frac{2}{\pi} \sqrt[3]{r^{2}-1}
$$

Moreover, $r^{*}=1$ is the unique zero of $f_{1}$. Thus, let $V$ be any open interval in $\left[r_{0}, r_{1}\right]$ containing $r^{*}$. Again, it is easy to construct a homotopy $\left\{g_{\sigma}: \bar{V} \rightarrow \mathbb{R}^{n} \mid \sigma \in[0,1]\right\}$ between $\left.f_{1}\right|_{\bar{V}}$ and $g_{1}: r \mapsto 1-r, r \in \bar{V}$, such that $0 \notin g_{\sigma}(\partial V), \forall \sigma \in[0,1]$ (see Figure 2).

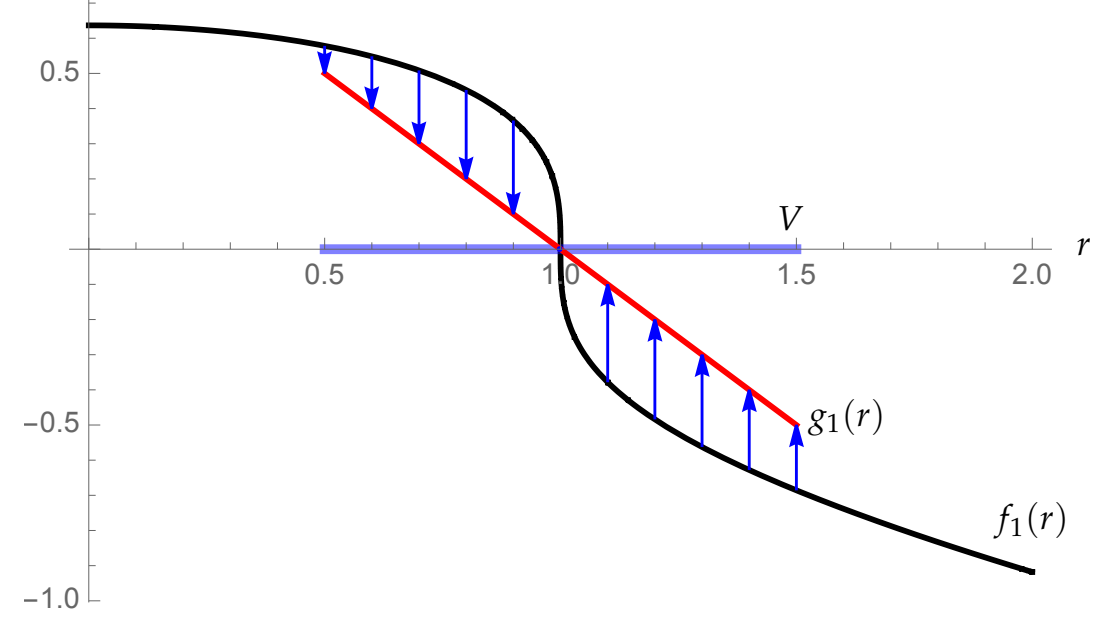

FIGURE 2. Illustration of the homotopy $g_{\sigma}$ between $f_{1}(r)$ and $g_{1}(r)=1-r$.

Again, Property P.3 of the Brouwer degree implies

$$
d_{B}\left(f_{1}, V, 0\right)=d_{B}\left(g_{1}, V, 0\right)=-1
$$

(see Remark 1). Hence, Theorem A guarantees, for $\varepsilon>0$ sufficiently small, the existence of a periodic solution $r(\theta, \varepsilon)$ of the differential equation (9) satisfying $r(\cdot, \varepsilon) \rightarrow r^{*}$, uniformly, as $\varepsilon \rightarrow 0$. This corresponds to a periodic solution $(x(t, \varepsilon), \dot{x}(t, \varepsilon))$ of the second order differential equation (8) satisfying $|(x(\cdot, \varepsilon), \dot{x}(\cdot, \varepsilon))| \rightarrow r^{*}$, uniformly, as $\varepsilon \rightarrow 0$.

Remark 2. The procedure employed in the examples above for computing explicitly the Brouwer degree of $\left(f_{1}, V, 0\right)$ always works for one-dimensional functions under similar suitable conditions, namely, if $a, b \in \mathbb{R}, a<b, V=[a, b]$, and $f: V \rightarrow \mathbb{R}$ is continuous with $f(a) f(b)<0$, then $d_{B}(f, V, 0)=\operatorname{sign}(f(b)-f(a))$.

\section{ACKNOWLEDGEMENTS}

The author thanks the referee for the constructive comments and suggestions which led to an improved version of the paper. 
DDN is partially supported by São Paulo Research Foundation (FAPESP) grants 2018/164308, 2018/ 13481-0, and 2019/10269-3, and by Conselho Nacional de Desenvolvimento Científico e Tecnológico (CNPq) grants 306649/2018-7 and 438975/ 2018-9.

\section{REFERENCES}

[1] N. N. Bogoliubov and Y. A. Mitropolsky. Asymptotic methods in the theory of non-linear oscillations. Translated from the second revised Russian edition. International Monographs on Advanced Mathematics and Physics. Hindustan Publishing Corp., Delhi, Gordon and Breach Science Publishers, New York, 1961.

[2] N. Bogolyubov. O Nekotoryh Statističeskih Metodah v Matematičeskoŭ Fizike (On Some Statistical Methods in Mathematical Physics). Akademiya Nauk Ukrainskoŭ SSR, Kiev, 1945.

[3] A. Buică and J. Llibre. Averaging methods for finding periodic orbits via Brouwer degree. Bulletin des sciences mathematiques, 128(1):7-22, 2004.

[4] A. Capietto, J. Mawhin, and F. Zanolin. Continuation theorems for periodic perturbations of autonomous systems. Trans. Amer. Math. Soc., 329(1):41-72, 1992.

[5] J. A. Cid, J. Mawhin, and M. Zima. An abstract averaging method with applications to differential equations. Journal of Differential Equations, 274:231-250, 2021.

[6] P. Fatou. Sur le mouvement d'un système soumis à des forces à courte période. Bull. Soc. Math. France, 56:98$139,1928$.

[7] R. Gaines and J. Mawhin. Coincidence Degree and Nonlinear Differential Equations. Lecture Notes in Mathematics. Springer Berlin Heidelberg, 1977.

[8] J. K. Hale. Ordinary differential equations. Robert E. Krieger Publishing Co., Inc., Huntington, N.Y., second edition, 1980.

[9] N. Krylov and N. Bogolyubov. Prilozhenie metodov nelineinoi mekhaniki k teorii statsionarnykh kolebanii (The Application of Methods of Nonlinear Mechanics to the Theory of Stationary oscillations). Kiev: Akademiya Nauk Ukrainskoı̆ SSR, Kiev], 1934.

[10] J. Kurzweil. Ordinary differential equations, volume 13 of Studies in Applied Mechanics. Elsevier Scientific Publishing Co., Amsterdam, 1986. Introduction to the theory of ordinary differential equations in the real domain, Translated from the Czech by Michal Basch.

[11] J. Llibre, A. C. Mereu, and D. D. Novaes. Averaging theory for discontinuous piecewise differential systems. J. Differential Equations, 258(11):4007-4032, 2015.

[12] J. Llibre, D. D. Novaes, and C. A. B. Rodrigues. Averaging theory at any order for computing limit cycles of discontinuous piecewise differential systems with many zones. Phys. D, 353/354:1-10, 2017.

[13] J. Llibre, D. D. Novaes, and M. A. Teixeira. Higher order averaging theory for finding periodic solutions via Brouwer degree. Nonlinearity, 27:563-583, 2014.

[14] J. Llibre, D. D. Novaes, and M. A. Teixeira. On the birth of limit cycles for non-smooth dynamical systems. Bull. Sci. Math., 139(3):229-244, 2015.

[15] J. Mawhin. Degré topologique et solutions périodiques des systèmes différentiels non linéaires. Bull. Soc. Roy. Sci. Liège, 38:308-398, 1969.

[16] J. Mawhin. Équations intégrales et solutions périodiques des systèmes différentiels non linéaires. Acad. Roy. Belg. Bull. Cl. Sci. (5), 55:934-947, 1969.

[17] J. Mawhin. Topological degree methods in nonlinear boundary value problems, volume 40 of CBMS Regional Conference Series in Mathematics. American Mathematical Society, Providence, R.I., 1979. Expository lectures from the CBMS Regional Conference held at Harvey Mudd College, Claremont, Calif., June 9-15, 1977.

[18] Y. A. Mitropolskii and N. V. Dao. Applied asymptotic methods in nonlinear oscillations, volume 55 of Solid Mechanics and its Applications. Kluwer Academic Publishers Group, Dordrecht, 1997.

[19] D. D. Novaes and F. B. Silva. Higher order analysis on the existence of periodic solutions in continuous differential equations via degree theory. SIAM Journal on Mathematical Analysis, 53(2):2476-2490, 2021.

[20] J. Sanders, F. Verhulst, and J. Murdock. Averaging Methods in Nonlinear Dynamical Systems. Applied Mathematical Sciences. Springer New York, 2007.

[21] F. Verhulst. Nonlinear differential equations and dynamical systems. Springer Science \& Business Media, 2006.

[22] E. Zeidler. Nonlinear functional analysis and its applications. I. Springer-Verlag, New York, 1986. Fixed-point theorems, Translated from the German by Peter R. Wadsack.

Departamento de Matemática - Instituto de Matemática, Estatística e ComputaÇão Científica (IMECC) - UNIVERSIDADE ESTADUAL DE CAMPINAS (UNICAMP), RUA SÉRGIO BUARQUE DE HOLANDA, 651, CidAdE UNIVERSITÁRIA ZEFERINO VAZ, 13083-859, CAMPINAS, SP, BRAZIL

Email address: ddnovaes@unicamp.br 